TRANSACTIONS OF THE

AMERICAN MATHEMATICAL SOCIETY

Volume 364, Number 11, November 2012, Pages 5589-5604

S 0002-9947(2012)05515-2

Article electronically published on June 8, 2012

\title{
TEST FUNCTIONS IN CONSTRAINED INTERPOLATION
}

\author{
MICHAEL A. DRITSCHEL AND JAMES PICKERING
}

\begin{abstract}
We give a set of test functions for $H_{1}^{\infty}$, the algebra of bounded holomorphic functions on the disk with first derivative equal to 0 , whose interpolation problem was studied by Davidson, Paulsen, Raghupathi and Singh (2009). We show that this set of test functions is minimal by relating these ideas to realization and interpolation problems.
\end{abstract}

\section{BACKGROUND AND INTRODUCTION}

There has recently been increased interest in constrained interpolation problems. These problems have many of the hallmarks of harder interpolation problems, in that they generally require collections of kernels, and have interesting behavior in the case of matrix-valued interpolation, much like interpolation on multiply connected domains. Nevertheless they are simple enough that we can do calculations explicitly (the kernels are typically rational functions), and we can re-use much of the theory of interpolation on the unit disk.

The basic problem, as discussed in [DPRS07], is the following: Under what circumstances can we find a bounded, holomorphic function $f$ on the disk, with zero derivative at 0 ; that is,

$$
f \in H_{1}^{\infty}:=\left\{g \in H^{\infty}: g^{\prime}(0)=0\right\},
$$

and which takes prescribed values $w_{1}, \ldots, w_{n}$ at prescribed points $z_{1}, \ldots, z_{n}$ ? The solution, as given in DPRS07, is analogous to the Nevanlinna-Pick theorem: such a function exists if and only if

$$
\left(\left(1-w_{i} \overline{w_{j}}\right) k^{s}\left(z_{j}, z_{i}\right)\right)_{i, j=1}^{n} \geq 0 \quad \forall s \in S^{2} .
$$

The kernels $k^{s}$ are in a particular class of kernels, parametrized by points $s$ on the real 2-sphere $S^{2}$. (They actually give two, different, Nevanlinna-Pick type theorems, but the second is not relevant here.)

In this paper, we look at interpolation with test functions in $H_{1}^{\infty}$. In a sense made clear in Section 3 , the test function approach to interpolation stands in duality to that of kernels. It originates with Agler. The test functions of a function algebra are certain elements which are assumed to be in the unit ball of the algebra. They determine a set of admissible kernels which in turn determine a norm for the algebra. The classic example is the function $f(z)=z$ on $\mathbb{D}$, which has as admissible kernels

Received by the editors April 21, 2009.

2010 Mathematics Subject Classification. Primary 47A57; Secondary 32C15, 46E20, 46E22, 47B32.

Key words and phrases. Interpolation, realizations, Nevanlinna-Pick, test functions.

This paper is based on work contributing to the second author's Ph.D. thesis, at the University of Newcastle-upon-Tyne, under the supervision of the first author. The work was funded in part by the Engineering and Physical Sciences Research Council.

(C)2012 American Mathematical Society Reverts to public domain 28 years from publication 
all those conjugate equivalent to the Szegö kernel, and which therefore gives rise to the normed function algebra which is $H^{\infty}(\mathbb{D})$.

The book by Agler and McCarthy AM02, in addition to the general background for the field, contains a treatment of Agler's approach to this subject and its application to realization and interpolation problems. A somewhat modified approach can be found in Dritschel and McCullough's paper DM07, from which we take our notation and a number of results.

There are circumstances (such as with $H^{\infty}(\mathbb{D})$ or $H^{\infty}\left(\mathbb{D}^{2}\right)$ ) where one can easily guess an optimal set of test functions. In most other situations a best choice is far from clear. There are a few cases (such as with $H^{\infty}$ on multiply connected planar domains) where optimal sets of test functions have been found ([DM05, DM07, Pic10]). The current paper adds yet another interesting example with $H_{1}^{\infty}$.

We determine a set of test functions for $H_{1}^{\infty}$, broadly following the approach of AHR08, via a Herglotz-type representation for $H_{1}^{\infty}$. These test functions turn out to be rational functions, and the set of test functions is parametrized by the sphere. We show, using techniques similar to those in [DM07, that our set of test functions is minimal. We give some indication of how these techniques could yield test functions for other types of constrained interpolation problems, although the theory appears to be less elegant in these situations.

Another key tool here is the notion of differentiating kernels. These are a simple analogue of reproducing kernels, and while they are not particularly interesting in and of themselves, they prove to be useful when working with problems of this sort.

\section{DifFEREntiating KERNELS}

In analogy with reproducing kernels, it is convenient to introduce what we call differentiating kernels. By Cauchy's integral formula, differentiation is a bounded linear functional on $H^{2}$, so for each $x \in \mathbb{D}$, and for each $i=0,1,2, \ldots$, there exists some function $k\left(x^{(i)}, \cdot\right) \in H^{2}$ such that

$$
f^{(i)}(x)=\left\langle f(\cdot), k\left(x^{(i)}, \cdot\right)\right\rangle .
$$

The above argument also holds for $H^{2}(R)$, for any finitely connected planar domain $R$. In the case of $H^{2}$ (that is, $H^{2}(\mathbb{D})$ ), by a straightforward calculation, we have explicitly that

$$
k\left(x^{(n)}, y\right)=n ! \frac{y^{n}}{(1-\bar{x} y)^{n+1}}=\frac{\partial^{n}}{\partial \bar{x}^{n}} k(x, y),
$$

where $k(x, y)=(1-\bar{x} y)^{-1}$ is the ordinary Szegö kernel. For brevity, we write $k_{x^{(i)}}$ for the function $k\left(x^{(i)}, \cdot\right)$.

If $M_{f}$ is the multiplication operator of $f$ on $H^{\infty}$, the differentiating kernels satisfy an analog of the reproducing kernel property

$$
M_{f}^{*} k_{x^{(n)}}=\sum_{j=0}^{n}\left(\begin{array}{l}
n \\
j
\end{array}\right) \overline{f^{(j)}(x)} k_{x^{(n-j)}},
$$


as

$$
\begin{aligned}
\left\langle g, M_{f}^{*} k_{x^{(n)}}\right\rangle & =\left\langle M_{f} g, k_{x^{(n)}}\right\rangle \\
& =\left\langle f g, k_{x^{(n)}}\right\rangle \\
& =(f g)^{(n)}(x) \\
& =\sum_{j=0}^{n}\left(\begin{array}{c}
n \\
j
\end{array}\right) f^{(j)}(x) g^{(n-j)}(x) \\
& =\left\langle g, \sum_{j=0}^{n}\left(\begin{array}{c}
n \\
j
\end{array}\right) \overline{f^{(j)}(x)} k_{x^{(n-j)}}\right\rangle .
\end{aligned}
$$

\section{Test FUnCtions}

3.1. Definitions. We refer the reader to DM07 for a more in-depth discussion of test functions, though many of the ideas are also covered in AM02. We follow the notation of [DM07]. For our purposes, we will need some basic definitions.

Definition 1. A set $\Psi$ of complex-valued functions on a set $X$ is a set of test functions if:

(1) For each $x \in X$,

$$
\sup \{|\psi(x)|: \psi \in \Psi\}<1 \text { and }
$$

(2) Whenever $F \subset X$ is a finite set with $n$ elements, the unital algebra generated by $\left.\Psi\right|_{F}$ (the restriction of $\Psi$ to $F$ ) is $n$-dimensional (that is, $\Psi$ separates points).

Definition 2. For any collection of test functions $\Psi$, we define a set of positive kernels, called the admissible kernels, as

$$
\mathcal{K}_{\Psi}:=\{k: X \times X \rightarrow \mathbb{C}:((1-\psi(x) \overline{\psi(y)}) k(x, y)) \geq 0 \forall \psi \in \Psi\} .
$$

We use the admissible kernels to define a normed algebra $H^{\infty}\left(\mathcal{K}_{\Psi}\right)$. We say a function $f: X \rightarrow \mathbb{C}$ is in $H^{\infty}\left(\mathcal{K}_{\Psi}\right)$ with $\|f\|_{H\left(\mathcal{K}_{\Psi}\right)} \leq 1$ if

$$
((1-f(x) \overline{f(y)}) k(x, y)) \geq 0 \forall k \in \mathcal{K}_{\Psi} .
$$

Since we have defined the unit ball of $H^{\infty}\left(\mathcal{K}_{\Psi}\right)$, by extension we have defined the whole of $H^{\infty}\left(\mathcal{K}_{\Psi}\right)$, and given its norm. Multiplication is defined pointwise.

While test functions can be used to construct interesting function algebras, for the purposes of this paper we want to reverse this procedure: given a normed function algebra $A$ over a set $X$, find a collection of test functions $\Psi$ such that $A=H^{\infty}\left(\mathcal{K}_{\Psi}\right)$.

This problem has a trivial solution; namely, take $\Psi$ to be the open unit ball of $A$. The problem becomes nontrivial when we impose some sort of minimality constraint on $\Psi$. For example, we may ask that no subset of $\Psi$ will suffice as a set of test functions for $A$. There is a difficulty with this though in that if $\Psi$ is not closed, it may always be possible to remove functions from $\Psi$ and still get a set of test functions for $A$. Consequently we also impose the constraint that $\Psi$ be a norm closed (and hence compact) subset of $A$.

Given a closed set of test functions $\Psi$ for $A=H^{\infty}\left(\mathcal{K}_{\Psi}\right)$, we can solve the so-called Agler-Pick interpolation problem. 
Theorem 3 ([DM07, Theorem 2.3 and its proof). Suppose $\Psi$ is a closed set of test functions. Let $F$, a finite subset of $X$, and $\xi: F \rightarrow \mathbb{D}$ be given.

(1) There exists $\varphi \in H^{\infty}\left(\mathcal{K}_{\Psi}\right)$ so that $\|\varphi\| \leq 1$ and $\left.\varphi\right|_{F}=\xi$.

(2) For each $k \in \mathcal{K}_{\Psi}$, the kernel

$$
F \times F \ni(x, y) \mapsto\left(1-\xi(x) \xi(y)^{*}\right) k(x, y)
$$

is positive.

(3) There exists a bounded positive measure $\mu$ on $\Psi$ and a positive kernel $\Gamma$ : $F \times F \rightarrow C(\Psi)^{*}$ so that for all $x, y \in F$,

$$
1-\xi(x) \xi(y)^{*}=\Gamma(x, y)\left(1-E(x) E(y)^{*}\right),
$$

where $E(x)$ is the multiplication operator on $L^{2}(\mu)$ given by $(E(x) f)(\psi)=$ $\psi(x) f(\psi)$ and $\Gamma(x, y)$ is $\mu$-absolutely continuous.

3.2. Zero mean probability measures. Our initial goal is to find a nonminimal set of test functions $\widehat{\Psi}$ for $H_{1}^{\infty}$.

Test functions have norm 1 or less, so the Möbius transform

$$
m: z \mapsto \frac{1+z}{1-z}
$$

(which takes the unit disk to the right half plane) takes $\psi \in \widehat{\Psi}$ to a function with positive real part. Since $H_{1}^{\infty} \subseteq H^{\infty}$, any test function $\psi$ has a Herglotz representation (see Theorem 1.1.19 of [AHR08]). So if $f(z)=m(\psi(z))$ with $f(0)>$ 0 , then

$$
f(z)=\int_{\mathbb{T}} \frac{w+z}{w-z} d \mu(w)
$$

for some positive measure $\mu$. As an element of $H_{1}^{\infty}, \psi^{\prime}(0)=0$. Since $m^{\prime} \neq 0$ and

$$
f^{\prime}(z)=\psi^{\prime}(z) m^{\prime}(\psi(z))
$$

it follows that $\psi^{\prime}(0)=0$ if and only if $f^{\prime}(0)=0$.

Now,

$$
f^{\prime}(z)=\int_{\mathbb{T}}\left(\frac{d}{d z} \frac{w+z}{w-z}\right) d \mu(w)=\int_{\mathbb{T}} \frac{2 w}{(w-z)^{2}} d \mu(w),
$$

so

$$
f^{\prime}(0)=\int_{\mathbb{T}} \frac{2}{w} d \mu(w)=2 \int_{\mathbb{T}} \bar{w} d \mu(w)=2 \overline{\int_{\mathbb{T}} w d \mu(w)} .
$$

If $\mu$ is a probability measure (which it will later be convenient to assume it is), then the condition that $\psi^{\prime}(0)=0$ is equivalent to the condition that $\mathbb{E}(\mu)=0$; that is, $\mu$ has zero-mean. It should be noted here that requiring $\mu$ to be a probability measure is equivalent to requiring that $f(0)=1$, or equivalently still, that $\psi(0)=0$.

We have proved the following:

Theorem 4. The analytic function $\psi$ has $\|\psi\|_{\infty} \leq 1, \psi(0)=0$, and $\psi^{\prime}(0)=0$ if and only if the corresponding measure is a zero-mean probability measure. 
3.3. Extreme directions. We also use techniques and definitions from [AHR08]. In that paper, they used the convention that if $A$ was a "real function space" in some sense, then $A^{h}$ is the set of all real functions in $A$ corresponding to (the real parts of) holomorphic functions. By analogy, we will write $A^{1}$ for the set of all real functions in $A$ corresponding to holomorphic functions with zero derivative at 0 . So, for example, if $L_{\mathbb{R}}^{2}(\mathbb{T})$ is defined in the usual way, then an element of $L_{\mathbb{R}}^{2,1}(\mathbb{T})$ is a function in $L_{\mathbb{R}}^{2}(\mathbb{T})$ which is the real part of an analytic function $f$ with $f^{\prime}(0)=0$. In this case it is easy to see that $\left(L_{\mathbb{R}}^{2,1}(\mathbb{T})\right)^{\perp}=\operatorname{span}\{\operatorname{Im} z, \operatorname{Re} z\}$, where $\perp$ denotes the annihilator. Consequently, $L_{\mathbb{R}}^{2,1}(\mathbb{T})$ has (real) co-dimension 2 in $L_{\mathbb{R}}^{2}(\mathbb{T})$.

We let $M_{\mathbb{R}}(\mathbb{T})$ be the space of finite regular real Borel measures on $\mathbb{T}$. By convention, we associate a harmonic function to a measure $\mu \in M_{\mathbb{R}}(\mathbb{T})$ via the Poisson kernel. Let $C_{\mathbb{R}}(\mathbb{T})$ be the space of real continuous functions on $\mathbb{T}$, so that $M_{\mathbb{R}}(\mathbb{T})$ is the dual of $C_{\mathbb{R}}(\mathbb{T})$, under the weak-* and uniform topologies, respectively. By our convention, we define real $M_{\mathbb{R}}^{1}(\mathbb{T})$ and $C_{\mathbb{R}}^{1}(\mathbb{T})$ as the subspaces of $M_{\mathbb{R}}(\mathbb{T})$ and $C_{\mathbb{R}}(\mathbb{T})$ (respectively), corresponding to holomorphic functions with zero derivative at 0 . Then, as in AHR08, we can see that

$$
M_{\mathbb{R}}^{1}(\mathbb{T})=\{\operatorname{Im} z, \operatorname{Re} z\}^{\perp}
$$

and

$$
C_{\mathbb{R}}^{1}(\mathbb{T})^{\perp}=\operatorname{span}\{\operatorname{Im} z d s, \operatorname{Re} z d s\} .
$$

We also need to make use of extreme directions. We say a nonzero vector $x$ in a cone $C$ is an extreme direction in $C$ if, for all $x_{1}, x_{2} \in C$ such that $x=x_{1}+x_{2}$, $x_{1}=t x$ and $x_{2}=s x$ for some $s, t \geq 0$.

This allows us to formulate the following:

Theorem 5. Let $E=\left\{\mu \in M_{\mathbb{R}}^{1}(\mathbb{T}): \mu \geq 0\right\}$. If $\mu$ is an extreme direction in $E$, then $\mu$ is supported at three or fewer points on $\mathbb{T}$.

Proof. Suppose $\mu$ is supported on four or more points in $\mathbb{T}$, and divide the support of $\mathbb{T}$ into four nonempty parts, $\Delta_{1}$ to $\Delta_{4}$. Let $\mu_{i}=\chi_{\Delta_{i}} \mu$, where $\chi_{\Delta_{i}}$ is the indicator function on $\Delta_{i}$. Let $\mathcal{M}=\operatorname{span}\left\{\mu_{1}, \ldots, \mu_{4}\right\}$. The dimension of $\mathcal{M}$ is 4 , and since $M_{\mathbb{R}}^{1}(\mathbb{T})$ has co-dimension 2 in $M_{\mathbb{R}}(\mathbb{T})$, we must have that

$$
\operatorname{dim}\left(\mathcal{M} \cap M_{\mathbb{R}}^{1}(\mathbb{T})\right) \geq 2 .
$$

Therefore, there exists a $\nu \in \mathcal{M} \cap M_{\mathbb{R}}^{1}(\mathbb{T})$ which is linearly independent of $\mu$. Since a measure $\alpha_{1} \mu_{1}+\cdots+\alpha_{4} \mu_{4} \in \mathcal{M}$ is positive whenever $\alpha_{1}, \ldots, \alpha_{4}$ are all positive, we can choose an $\epsilon>0$ small enough that $\mu \pm \epsilon \nu \geq 0$. Hence, $\frac{1}{2}(\mu \pm \epsilon \nu) \in E$, but

$$
\mu=\frac{1}{2}(\mu+\epsilon \nu)+\frac{1}{2}(\mu-\epsilon \nu),
$$

so $\mu$ is not an extreme direction in $E$.

We can combine this with Theorem 4, which says that $\mathbb{E}(\mu)=0$ for $\mu \in E$ :

- If $\mu$ is supported at one point of $\mathbb{T}$, then it is clearly impossible to have $\mathbb{E}(\mu)=0$.

- If $\mu$ is supported at two points, then 0 must be in the convex hull of these two points (the line between them), and so the two points must lie opposite each other on the circle, and both have equal weight (if $\mu$ is to be a probability measure, this weight must be $\frac{1}{2}$ ). 

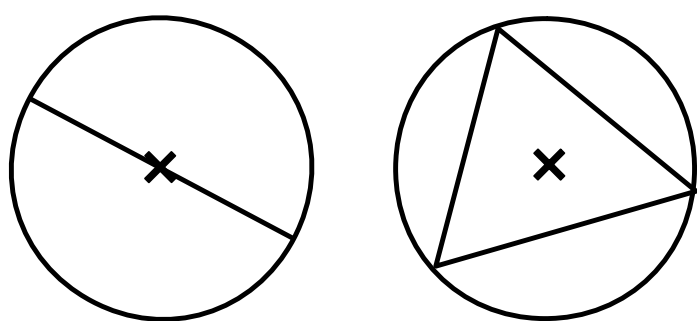

Figure 3.1. Types of elements in $\widehat{\Theta}$

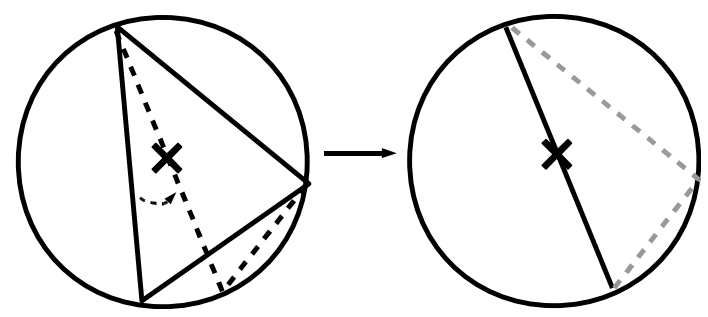

FiguRE 3.2. Convergence in $\widehat{\Theta}$

- If $\mu$ is supported at three points, then these points must be such that 0 is in the interior of their convex hull (that is, the interior of the triangle they form; if 0 lies on one of the lines of the triangle, then $\mu$ will only be supported on the two points at either end of the line, so this is the degenerate case we had before). We can also see that if $\mu$ is a probability measure, then its weights are uniquely determined by its support - the weights are precisely the barycentric co-ordinates of 0 , with respect to the three vertices of the triangle.

As a consequence, it is easy to see that, if a measure $\mu$ in $E$ is supported on three or fewer points, it is uniquely determined by those points, up to multiplication by a scalar. In particular, if $\mu=t_{1} \mu_{1}+t_{2} \mu_{2}$, for some $\mu_{1}, \mu_{2} \in E$, then $\mu_{1}$ and $\mu_{2}$ must be supported on a subset of the support of $\mu$, so must be scalar multiples of $\mu$. Thus we have characterized the extreme directions in $E$. By assumption, extreme directions are nonzero, and any nonzero measure in $E$ can be scaled to a probability measure.

3.4. From extreme directions to test functions. We use $\widehat{\Theta}$ to denote the set of zero-mean probability measures which are extreme directions in $E$. As we observed above, such measures are either supported

- at the endpoints of diameters of the circle;

- on vertices of triangles, where the vertices lie on the circumference of the circle, and the center of the circle is in the interior.

(See Figure 3.1) We endow $\widehat{\Theta}$ with the relative weak-* topology coming from $M_{\mathbb{R}}(\mathbb{T})$. Consequently, a sequence of measures in $\widehat{\Theta}$ converges if and only if the corresponding sequence of vertices converges pointwise. If a sequence of (vertices of) triangles happens to converge to a triangle where one side is a diameter, then the weight of the third vertex will tend to zero, as in Figure 3.2 . 


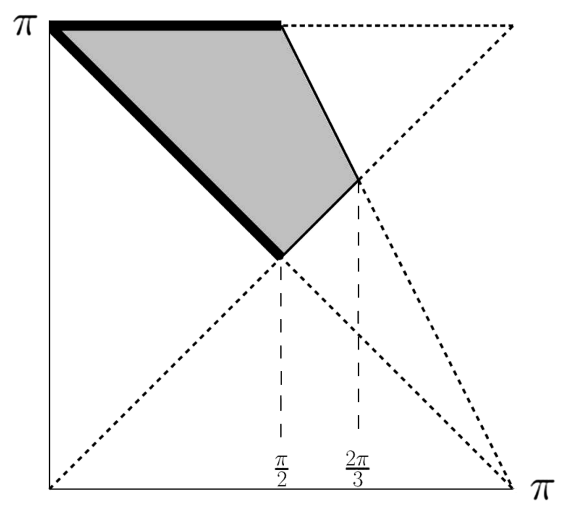

FiguRE 3.3. Allowed values of $\left(\theta_{1}, \theta_{2}\right)$ for measures in $\Theta$

As we will see later, if $\mu$ is a zero-mean probability measure supported on $n$ points, then $\mu$ gives rise to a test function $\psi_{\mu}$, which will be an $n$-to-one Blaschke product, with $\psi_{\mu}^{\prime}(0)=0, \psi_{\mu}(0)=0$, and $\psi_{\mu}(w)=1$ precisely when $w$ is in the support of $\mu$. Conversely, if we have such a function $\psi$, then there is a corresponding zero-mean probability measure $\mu_{\psi}$, with support $\psi^{-1}(\{1\})$.

If we have a test function $\psi_{\mu}$, corresponding to a $\mu \in \widehat{\Theta}$, then $\widetilde{\psi}:=\overline{\psi_{\mu}(1)} \psi_{\mu}$ is a two-or-three-to-one inner function of the type required, and since $\widetilde{\psi}(1)=1$, the corresponding measure $\widetilde{\mu}$ is supported at 1 . This is useful, as we can identify two test functions if one is a unimodular constant multiple of the other.

In light of this, we define an equivalence relation $\sim$ on $\widehat{\Theta}$ by

$$
\mu_{1} \sim \mu_{2} \Longleftrightarrow \psi_{\mu_{1}}=\lambda \psi_{\mu_{2}} \text { for some }\|\lambda\|=1 .
$$

Then we can define $\Theta:=\widehat{\Theta} / \sim$. By the above reasoning, elements of $\Theta$ are uniquely identified with elements of a certain set triangles or diameters in $\widehat{\Theta}$ with a vertex/endpoint at 1 (i.e., on the rightmost point of the circle). When viewed in this way, there is only one diameter in $\Theta$.

It is interesting to note that $\Theta$ is homeomorphic to $S^{2}$. We sketch a proof. Let $\theta_{1}, \theta_{2}, \theta_{3}$ represent the three angles formed by the radii ending in the three triangle vertices (where we take two of the vertices to be the same in the degenerate case of a diameter), ordered counterclockwise from the positive real axis. In order for the corresponding triangle to contain the origin, all angles must be less than or equal to $\pi$. By rotating the triangle if necessary, we may assume without loss of generality that $\theta_{1}$ is the smallest angle. Furthermore, $\theta_{3}=2 \pi-\theta_{1}-\theta_{2}$, so each triangle (and hence the corresponding element of $\Theta$ ) is uniquely determined by specifying $\theta_{1}$ and $\theta_{2}$. We therefore have four constraints,

$$
\begin{aligned}
\theta_{2} \leq \pi, & \theta_{2} \geq \pi-\theta_{1}, \\
\theta_{2} \leq 2 \pi-2 \theta_{1}, & \theta_{2} \geq \theta_{1},
\end{aligned}
$$

which bound the shaded region in Figure 3.3. Equality in the top two constraints corresponds to either $\theta_{2}=\pi$ or $\theta_{3}=\pi$ (indicated in the figure by the heavier boundary segments), and so are identified with the unique diagonal. For each $\frac{\pi}{2} \leq \theta_{1} \leq \frac{2 \pi}{3}$, we identify the points on the boundary of the region (on the lighter segments), since these correspond to $\theta_{3}=\theta_{1}$ and $\theta_{2}=\theta_{1}$, respectively, and the 
associated triangles are equivalent after rotation. After making these identifications, no two points in the shaded region correspond to rotationally equivalent triangles, and clearly the region is homeomorphic to $S^{2}$.

3.5. The test functions. The set of probability measures in $M_{\mathbb{R}}^{1}(\mathbb{T})$ is a subset of $E$, and in fact corresponds to $E_{\rho}$, in the sense of Lemma 3.4 of [AHR08], where $\rho(\mu)=\mu(\mathbb{T})$. We know, therefore, that $E_{\rho}$ is convex, and we showed above that its extreme points are given by $\widehat{\Theta}$. The set $E_{\rho}$ is compact by the Banach-Alaoglu theorem, so by the Choquet-Bishop-de Leeuw theorem, for any $\mu \subseteq E_{\rho}$, there exists a probability measure $\nu_{\mu}$ on $\widehat{\Theta}$ such that

$$
\mu=\int_{\widehat{\Theta}} \vartheta d \nu_{\mu}(\vartheta) .
$$

By the Herglotz representation theorem, probability measures in $M_{\mathbb{R}}^{1}(\mathbb{T})$ correspond to analytic functions $f$ on $\mathbb{D}$ with positive real part, $f^{\prime}(0)=0$, and $f(0)=1$. For all $\vartheta \in \widehat{\Theta}$, define

$$
h_{\vartheta}(z):=\int_{\mathbb{T}} \frac{w+z}{w-z} d \vartheta(w)
$$

and

$$
\psi_{\vartheta}=\frac{h_{\vartheta}-1}{h_{\vartheta}+1} .
$$

Then reasoning as above, we can write

$$
\begin{aligned}
f(z) & =\int_{\mathbb{T}} \frac{w+z}{w-z} d \mu(w) \\
& =\int_{\mathbb{T}} \frac{w+z}{w-z}\left[\int_{\widehat{\Theta}}(d \vartheta(w)) d \nu_{\mu}(\vartheta)\right] \\
& =\int_{\widehat{\Theta}}\left[\int_{\mathbb{T}} \frac{w+z}{w-z} d \vartheta(w)\right] d \nu_{\mu}(\vartheta) \\
& =\int_{\widehat{\Theta}} h_{\vartheta}(z) d \nu_{\mu}(\vartheta) .
\end{aligned}
$$

This gives us a new Herglotz-type representation, which we call an Agler-Herglotz representation:

Theorem 6. If $f$ is an analytic function on $\mathbb{D}$ with positive real part, $f^{\prime}(0)=0$, and $f(0)>1$, then there exists some positive real measure $\nu$ on $\widehat{\Theta}$ such that

$$
f(z)=\int_{\widehat{\Theta}} h_{\vartheta}(z) d \nu(\vartheta) .
$$

We now prove the main result; namely, that $\Psi:=\left\{\psi_{\vartheta}: \vartheta \in \Theta\right\}$ is a set of test functions for $H_{1}^{\infty}(\mathbb{D})$.

Theorem 7. The two spaces $H^{\infty}\left(\mathcal{K}_{\Psi}\right)$ and $H_{1}^{\infty}(\mathbb{D})$ are isometrically isomorphic, that is, $H^{\infty}\left(\mathcal{K}_{\Psi}\right)=H_{1}^{\infty}(\mathbb{D})$ as algebras and $\|\cdot\|_{\mathcal{K}_{\Psi}}=\|\cdot\|_{H_{1}^{\infty}(\mathbb{D})} \cdot$

Proof. One way is simple. Since $\Psi \subseteq H_{1}^{\infty}(\mathbb{D})$, it follows that $\mathcal{K}_{\Psi}$ contains the set $\mathcal{K}_{1}^{\infty}$ of reproducing kernels given in [DPRS07]. So if $\zeta \in H^{\infty}\left(\mathcal{K}_{\Psi}\right)$, and $\|\zeta\|_{\mathcal{K}_{\Psi}} \leq 1$, then

$$
([1-\zeta(z) \overline{\zeta(w)}] k(x, w)) \geq 0
$$


for all $k \in \mathcal{K}_{1}^{\infty}$. Therefore, $\zeta$ must be in $H_{1}^{\infty}(\mathbb{D})$, with $\|\zeta\|_{H_{1}^{\infty}(\mathbb{D})} \leq 1$, and so $H^{\infty}\left(\mathcal{K}_{\Psi}\right) \subseteq H_{1}^{\infty}(\mathbb{D})$.

Now, suppose conversely that $\zeta \in H_{1}^{\infty}(\mathbb{D})$ and $\|\zeta\|_{H_{1}^{\infty}(\mathbb{D})} \leq 1$. For now, we also suppose that $\zeta(0)=0$. Let

$$
f:=\frac{1+\zeta}{1-\zeta}
$$

so

$$
\zeta=\frac{f-1}{f+1}
$$

Hence

$$
1-\zeta(z) \overline{\zeta(w)}=2 \frac{f(z)+\overline{f(w)}}{(f(z)+1)(\overline{f(w)}+1)} .
$$

The function $f$ has positive real part, $f(0)=1$ and $f^{\prime}(0)=0$, so by the AglerHerglotz representation and Theorem [6, there is a measure $\nu$ on $\widehat{\Theta}$ such that

$$
f=\int_{\widehat{\Theta}} h_{\vartheta} d \nu(\vartheta) \text {. }
$$

Using the definition of $\psi_{\vartheta}$ and (3.1), we find that

$$
1-\zeta(z) \overline{\zeta(w)}=\int_{\widehat{\Theta}} \frac{1-\psi_{\vartheta}(z) \overline{\psi_{\vartheta}(w)}}{(f(z)+1)\left(1-\psi_{\vartheta}(z)\right)\left(1-\overline{\psi_{\vartheta}(w)}\right)(\overline{f(w)}+1)} d \nu(\vartheta)
$$

Recall that if $\vartheta \in \widehat{\Theta}$, then $\overline{\psi_{\vartheta}(1)} \psi_{\vartheta} \in \Psi$. Define a positive kernel $\Gamma: \mathbb{D} \times \mathbb{D} \rightarrow C_{b}(\Psi)^{*}$ by

$$
\Gamma(z, w) \alpha=\int_{\widehat{\Theta}} \frac{\alpha\left(\overline{\psi_{\vartheta}(-1)} \psi_{\vartheta}\right)}{(f(z)+1)\left(1-\psi_{\vartheta}(z)\right)\left(1-\overline{\psi_{\vartheta}(w)}\right)(\overline{f(w)}+1)} d \nu(\vartheta),
$$

where $\alpha \in C_{b}(\Psi)$. Then

$$
1-\zeta(z) \overline{\zeta(w)}=\Gamma(z, w)\left(1-E(z) E(w)^{*}\right),
$$

and so $\zeta \in H^{\infty}\left(\mathcal{K}_{\Psi}\right)$ with $\|\zeta\|_{\mathcal{K}_{\Theta}} \leq 1$.

The same result holds when $\zeta(0) \neq 0$, since

$$
1-\left(\frac{\zeta(z)-a}{1-\bar{a} \zeta(z)}\right) \overline{\left(\frac{\zeta(w)-a}{1-\bar{a} \zeta(w)}\right)}=\frac{(1-a \bar{a})(1-\zeta(z) \overline{\zeta(w)})}{(1-\bar{a} \zeta(z))(1-a \overline{\zeta(w)})} .
$$

Therefore in all cases, $\zeta \in H^{\infty}\left(\mathcal{K}_{\Psi}\right)$, and $\|\zeta\|_{\mathcal{K}_{\Psi}} \leq 1$, so $H^{\infty}\left(\mathcal{K}_{\Psi}\right)=H_{1}^{\infty}(\mathbb{D})$ and $\|\cdot\|_{\mathcal{K}_{\Psi}}=\|\cdot\|_{H_{1}^{\infty}(\mathbb{D})}$, as required.

3.6. Minimality. Following [DM07, we show that the set of test functions $\Psi$ is minimal, in the sense that there is no proper closed subset $C \subset \Psi$ so that $C$ is a set of test functions for $H_{1}^{\infty}$. The proof requires the following lemma.

Lemma 8. Let $\mu$ be a measure on a space $C, H$ a separable Hilbert space, and $f \in B(H) \otimes L^{1}(\mu)$ with $f(x) \geq 0 \mu$-almost everywhere. If for some $M \in B(H)$, $M \geq \int_{C} f(x) d \mu(x)$, then for all $\delta>0$ there exists some $C_{\delta} \subseteq C$ and $N_{\delta}>0$, such that $\mu\left(C-C_{\delta}\right)<\delta$ and $M \geq N_{\delta} f(x)$ for all $x \in C_{\delta}$. 
Proof. Define, for $N>0$, and $\varphi \in H$,

$$
\begin{gathered}
C_{N}^{\varphi}=\{x \in C:\langle(M-N f(x)) \varphi, \varphi\rangle \geq 0\}, \\
C_{N}=\{x \in C: M \geq N f(x)\}, \\
C_{0}=\bigcup_{N>0} C_{N}, \quad C_{0}^{\varphi}=\bigcup_{N>0} C_{N}^{\varphi} .
\end{gathered}
$$

For any given $\varphi \in H, C-C_{0}^{\varphi}$ is a $\mu$-null set. To see this, note that for $x$ to be in $C-C_{0}^{\varphi}$, we would need to have $\langle M \varphi, \varphi\rangle=0$ but $\langle f(x) \varphi, \varphi\rangle>0$. However,

$$
\langle M \varphi, \varphi\rangle \geq\left\langle\int_{C} f(x) d \mu(x) \varphi, \varphi\right\rangle \geq \int_{C-C_{0}^{\varphi}}\langle f(x) \varphi, \varphi\rangle d \mu(x)>0,
$$

which is a contradiction.

If $\Phi$ is a countable dense subset of the unit ball in $H$, then

$$
C_{N}=\bigcap_{\varphi \in \Phi} C_{N}^{\varphi}
$$

and so

$$
\begin{aligned}
C-C_{0} & =\bigcap_{N>0}\left(C-C_{N}\right)=\bigcap_{N>0}\left(\bigcup_{\varphi \in \Phi}\left[C-C_{N}^{\varphi}\right]\right) \\
& =\bigcup_{\varphi \in \Phi}\left(\bigcap_{N>0}\left[C-C_{N}^{\varphi}\right]\right)=\bigcup_{\varphi \in \Phi}\left(C-C_{0}^{\varphi}\right),
\end{aligned}
$$

which is a countable union of null sets, and so is a null set. Consequently, as $N \rightarrow 0$, $\mu\left(C-C_{N}\right) \rightarrow 0$, and $M \geq N f(x)$ for all $x \in C_{N}$, concluding the proof.

Theorem 9. No proper closed subset $C$ of $\Psi$ is a set of test functions for $H_{1}^{\infty}$.

Proof. Suppose, towards an eventual contradiction, that $C$ is a proper closed subset of $\Psi$ and $\psi_{0}=\psi_{\vartheta_{0}} \notin C$. Since $C$ is closed, its complement is open, so we can safely assume that $\vartheta_{0}$ is not supported at the endpoints of a diameter, or vertices of an equilateral triangle (these cases corresponding to the test functions $z^{2}$ and $z^{3}$ ).

The differentiating kernels defined in Section 2 are rational functions, so we can extend them to the entire Riemann sphere, $\mathbb{C} \cup\{\infty\}$. Upon doing so, if $x \neq 0, k_{x^{(n)}}$ has $n+1$ poles, all at $\overline{x^{-1}}$, and $k_{0^{(n)}}$ has $n$ poles, all at $\infty$.

The kernels

$$
\Delta_{\vartheta}(z, w):=\left(1-\psi_{\vartheta}(z) \overline{\psi_{\vartheta}(w)}\right) k(w, z)
$$

are positive and have rank at most three (here $k$ is the Szegö kernel). To see this, first note that since the diameter case is excluded, $\psi_{\vartheta}$ has precisely three zeroes, and that at least two of them must be at zero, as $\psi_{\vartheta}(0)=0$ and $\psi_{\vartheta}^{\prime}(0)=0$. Also note that $M_{\vartheta}$, the operator of multiplication by $\psi_{\vartheta}$, is an isometry on $H^{2}$, so $1-M_{\vartheta} M_{\vartheta}^{*}$ is the projection onto

$$
\mathfrak{M}_{\vartheta}:=\operatorname{ker} M_{\vartheta}^{*}=\operatorname{Span}\left\{k_{0}, k_{0^{\prime}}, k_{a_{\vartheta}}\right\},
$$

where $a_{\vartheta}$ is the third zero of $\vartheta$ (if $\vartheta$ has three zeroes at zero, then $a_{\vartheta}=0^{\prime \prime}$ ).

Now,

$$
\Delta_{\vartheta}(z, w)=\left\langle\left(1-M_{\vartheta} M_{\vartheta}^{*}\right) k_{w}, k_{z}\right\rangle:=\left\langle P_{\vartheta} k_{w}, k_{z}\right\rangle=\left\langle P_{\vartheta} k_{w}, P_{\vartheta} k_{z}\right\rangle
$$


is a holomorphic function in $z$ and an anti-holomorphic function in $w$. If we think of the anti-holomorphic function as being in the dual of $H^{2}$, then

$$
\Delta_{\vartheta} \in H^{2} \otimes\left(H^{2}\right)^{*} \cong B\left(H^{2}\right) .
$$

More explicitly, $\Delta_{\vartheta}$ defines an operator on $H^{2}$ as

$$
\Delta_{\vartheta} f(z):=\int_{\mathbb{T}} \Delta_{\vartheta}(z, w) f(w) d s(w),
$$

and so

$$
\begin{aligned}
\left\langle\Delta_{\vartheta} f, g\right\rangle & =\int_{\mathbb{T}} \int_{\mathbb{T}} \overline{g(z)} \Delta_{\vartheta}(z, w) f(w) d s(w) d s(z) \\
& =\int_{\mathbb{T}} \int_{\mathbb{T}} \overline{g(z)}\left\langle P_{\vartheta} k_{w}, P_{\vartheta} k_{z}\right\rangle f(w) d s(w) d s(z) \\
& =\int_{\mathbb{T}} \int_{\mathbb{T}}\left\langle f(w) P_{\vartheta} k_{w}, g(z) P_{\vartheta} k_{z}\right\rangle d s(w) d s(z) \\
& =\left\langle\int_{\mathbb{T}} f(w) P_{\vartheta} k_{w} d s(w), \int_{\mathbb{T}} g(z) P_{\vartheta} k_{z} d s(z)\right\rangle \\
& =\left\langle\int_{\mathbb{T}} f(w) P_{\vartheta} k_{w} d s(w), \int_{\mathbb{T}} g(z) P_{\vartheta} k_{z} d s(z)\right\rangle_{\mathfrak{M}_{\vartheta}}
\end{aligned}
$$

Hence, we have factored $\Delta_{\vartheta}$ as $A_{\vartheta}^{*} A_{\vartheta}$, where $A_{\vartheta}: H^{2} \rightarrow \mathfrak{M}_{\vartheta}$ is given by

$$
A_{\vartheta} f:=\int_{\mathbb{T}} f(w) P_{\vartheta} k_{w} d s(w) .
$$

We also note now, for use later, that $A_{\vartheta}^{*}=I_{\mathfrak{M}_{\vartheta}}$, the embedding map of $\mathfrak{M}_{\vartheta}$ into $H^{2}$, as

$$
\begin{aligned}
\left\langle A_{\vartheta} f, g\right\rangle & =\left\langle\int_{\mathbb{T}} f(w) P_{\vartheta} k_{w} d s(w), g\right\rangle_{\mathfrak{M}_{\vartheta}} \\
& =\int_{\mathbb{T}} f(w)\left\langle P_{\vartheta} k_{w}, g\right\rangle_{\mathfrak{M}_{\vartheta}} d s(w) \\
& =\int_{\mathbb{T}} f(w)\left\langle P_{\vartheta} k_{w}, I_{\mathfrak{M}_{\vartheta}} g\right\rangle d s(w) \\
& =\int_{\mathbb{T}} f(w)\left\langle k_{w}, I_{\mathfrak{M}_{\vartheta}} g\right\rangle d s(w) \\
& =\int_{\mathbb{T}} f(w) \overline{\left(I_{\mathfrak{M}_{\vartheta}} g\right)(w)} d s \\
& =\left\langle f, I_{\mathfrak{M}_{\vartheta}} g\right\rangle .
\end{aligned}
$$

We choose any set of four points $F=\left\{z_{1}, z_{2}, z_{3}, z_{4}\right\} \in \mathbb{D}$, and consider the classical Nevanlinna-Pick problem of finding a contractive function $\varphi \in H^{\infty}$ such that $\varphi\left(z_{i}\right)=\psi_{0}\left(z_{i}\right)$ for all $i$. Since $\Delta_{0}(z, w)$ has rank at most three, the $4 \times 4$ matrix

$$
\left(\left[1-\psi_{0}\left(z_{i}\right) \overline{\psi_{0}\left(z_{j}\right)}\right] k\left(z_{j}, z_{i}\right)\right)_{i, j=1}^{4}
$$

must be singular, so the problem has a unique solution; namely $\varphi=\psi_{0}$.

Assuming that $C$ is a set of test functions for $H_{1}^{\infty}$, then by Theorem 2.3 of DM07. (see Theorem 3 above), there must be a positive kernel $\Gamma: F \times F \rightarrow C(C)^{*}$ 
such that

$$
1-\psi_{0}\left(z_{i}\right) \overline{\psi_{0}\left(z_{j}\right)}=\Gamma\left(z_{i}, z_{j}\right)\left(1-E\left(z_{i}\right) E\left(z_{j}\right)^{*}\right) .
$$

Indeed, by Theorem 2.2 of $\mathrm{DM} 07$, this kernel extends to the whole of $\mathbb{D} \times \mathbb{D}$. We can rewrite this, in our case, by saying that there exists a measure $\mu$ on $C$, and functions $h_{l}(z, \cdot) \in L^{2}(\mu)$, for $l=1, \ldots, 4$, such that

$$
1-\psi_{0}(z) \overline{\psi_{0}(w)}=\int_{C} \sum_{l=1}^{4} h_{l}(z, \psi) \overline{h_{l}(w, \psi)}(1-\psi(z) \overline{\psi(w)}) d \mu(\psi)
$$

Multiplying this equation by $k(z, w)$ gives

$$
\Delta_{0}(z, w)=\int_{C} \sum_{l=1}^{4} h_{l}(z, \psi) \Delta_{\psi}(z, w) \overline{h_{l}(w, \psi)} d \mu(\psi) .
$$

Since $\Delta_{\psi}$ is a positive kernel and a positive operator, when seen as an operator on $H^{2}$, as above, we have that for all $l$,

$$
\Delta_{0}(z, w) \geq \int_{C} h_{l}(z, \psi) \Delta_{\psi}(z, w) \overline{h_{l}(w, \psi)} d \mu(\psi) .
$$

By Lemma 8, for any $\delta>0$, there is a set $C_{\delta}$, and a constant $c_{\delta}>0$ such that $\mu\left(C-C_{\delta}\right)<\delta$, and

$$
\Delta_{0}(z, w) \geq c_{\delta} h_{l}(z, \psi) \Delta_{\psi}(z, w) \overline{h_{l}(w, \psi)}
$$

for all $\psi \in C_{\delta}$.

If we use the factorization of $\Delta_{\vartheta}$ from above, we have

$$
A_{0}^{*} A_{0} \geq c_{\delta} h_{l}(z, \psi) A_{\psi}^{*} A_{\psi} \overline{h_{l}(w, \psi)},
$$

and so by Douglas' Lemma, the range of $h_{l}(\cdot, \psi) A_{\psi}^{*}$ is contained in the range of $A_{0}^{*}$. Therefore, there exist constants $c_{1}, \ldots, c_{9}$ so that

$$
\begin{aligned}
h_{l}(\cdot, \psi) k_{0} & =c_{1} k_{0}+c_{2} k_{0^{\prime}}+c_{3} k_{a_{0}}, \\
h_{l}(\cdot, \psi) k_{0^{\prime}} & =c_{4} k_{0}+c_{5} k_{0^{\prime}}+c_{6} k_{a_{0}}, \\
h_{l}(\cdot, \psi) k_{a_{\psi}} & =c_{7} k_{0}+c_{8} k_{0^{\prime}}+c_{9} k_{a_{0}} .
\end{aligned}
$$

By letting $\delta$ go to zero, we see that these equations must hold for $\mu$-almost-all $\psi \in C$.

By (3.3),$h_{l}(\cdot, \psi)=c_{1} k_{0}+c_{2} k_{0^{\prime}}+c_{3} k_{a_{0}}$ for appropriate constants $c_{1}, c_{2}$ and $c_{3}$, as $k_{0}$ is constant. We find that $h_{l}(\cdot, \psi)$ must extend meromorphically to the Riemann sphere, as these kernels do so. Equation (3.4) implies that $c_{2}=0$, as otherwise the left hand side of the equation has a triple pole at $\infty$, while the right hand side has at most a double pole.

We consider equation (3.5) in three cases. First, if $\psi$ has only two zeroes, there is no equation (3.5), so $h_{l}(\cdot, \psi)=c_{1}+c_{3} k_{a_{0}}$. 
If $a_{\psi} \neq 0$, then

$$
\begin{aligned}
h_{l}(y, \psi) k_{a_{\psi}}(y)= & c_{7} k_{0}(y)+c_{8} k_{0^{\prime}}(y)+c_{9} k_{a_{0}}(y) \\
{\left[c_{1}+c_{3} \frac{1}{1-\overline{a_{0}} y}\right] \frac{1}{1-\overline{a_{\psi}} y}=} & c_{7}+c_{8} y+c_{9} \frac{1}{1-\overline{a_{0}} y}, \\
c_{1}+c_{3} \frac{1}{1-\overline{a_{0}} y}= & c_{7}-c_{7} \overline{a_{\psi}} y+c_{8} y-c_{8} \overline{a_{\psi}} y^{2}+\frac{c_{9}-c_{9} \overline{a_{\psi}} y}{1-\overline{a_{0}} y} \\
c_{1}-c_{1} \overline{a_{0}} y+c_{3}= & c_{7}-c_{7} \overline{a_{\psi}} y-c_{7} \overline{a_{0}} y+c_{7} \overline{a_{\psi} a_{0}} y^{2} \\
& +c_{8} y-c_{8} \overline{a_{\psi}} y^{2}-c_{8} \overline{a_{0}} y^{2}+c_{8} \overline{a_{\psi} a_{0}} y^{3} \\
& +c_{9}-c_{9} \overline{a_{\psi}} y .
\end{aligned}
$$

Looking at the $y^{3}$ term, we see that $c_{8}=0$, then looking at the $y^{2}$ term we see that $c_{7}=0$, and finally comparing the constant and $y$ coefficients, we have

$$
\begin{gathered}
\left\{\begin{array}{c}
c_{1}+c_{3}=c_{9} \\
c_{1} \overline{a_{0}}=c_{9} \overline{a_{\psi}}
\end{array}\right. \\
\left\{\begin{array}{l}
c_{1}=c_{9} \overline{\overline{q_{\psi}}} \\
c_{3}=c_{9}\left(1-\frac{\overline{a_{\psi}}}{\overline{a_{0}}}\right)
\end{array}\right.
\end{gathered}
$$

and so

$$
h_{l}(y, \psi)=c_{9}\left(\frac{\overline{a_{\psi}}}{\overline{a_{0}}}+\left(1-\frac{\overline{a_{\psi}}}{\overline{a_{0}}}\right) \frac{1}{1-\overline{a_{0} y}}\right)=c \frac{1-\overline{a_{\psi}} y}{1-\overline{a_{0}} y} .
$$

Alternately, if $a_{\psi}=0$, then equation (3.5) becomes

$$
\begin{aligned}
& h_{l}(y, \psi) y^{2}=c_{7}+c_{8} y+c_{9} \frac{1}{1-\overline{a_{0}} y}, \\
& c_{1} y^{2}+c_{3} \frac{y^{2}}{1-\overline{a_{0}} y}=c_{7}+c_{8} y+c_{9} \frac{1}{1-\overline{a_{0}} y}, \\
& c_{1} y^{2}-c_{1} \overline{a_{0}} y^{3}+c_{3} y^{2}=c_{7}+c_{7} \overline{a_{0}} y+c_{8} y+c_{8} \overline{a_{0}} y^{2}+c_{9} .
\end{aligned}
$$

Considering the $y^{3}$ term we see that $c_{1} \overline{a_{0}}=0$, so $c_{1}=0$. The $y^{2}$ terms then lead us to conclude that $c_{3}=c_{8} \overline{a_{0}}$. Consequently,

$$
h_{l}(y, \psi)=c_{3} \frac{1}{1-\overline{a_{0}} y}=c \frac{1-\overline{a_{\psi}} y}{1-\overline{a_{0}} y},
$$

as before.

Combining these consequences of equations (3.3)-(3.5) with (3.2) gives a more explicit realization than the one in (3.2); namely,

$$
\begin{aligned}
1-\psi_{0}(z) \overline{\psi_{0}(w)}=\sum_{l=1}^{4} & \left(\alpha_{l}+\frac{\beta_{l}}{1-\overline{a_{0}} z}\right)\left(\overline{\alpha_{l}}+\frac{\overline{\beta_{l}}}{1-a_{0} \bar{w}}\right)\left(1-\psi_{\infty}(z) \overline{\psi_{\infty}(w)}\right) \\
& +\int_{C} c(\psi)\left(\frac{1-\overline{a_{\psi}} z}{1-\overline{a_{0}} z}\right)\left(\frac{1-a_{\psi} \bar{w}}{1-a_{0} \bar{w}}\right)(1-\psi(z) \overline{\psi(w)}) d \mu(\psi)
\end{aligned}
$$


for some positive $c \in L^{1}(\mu)$ and some $\alpha_{1}, \beta_{1}, \ldots, a_{4}, \beta_{4} \in \mathbb{C}$. The $\psi$ s are Blaschke products, and we know their roots, so we can write this even more explicitly as

$$
\begin{array}{r}
1-z^{2} \bar{w}^{2} \frac{z-a_{0}}{1-\overline{a_{0}} z} \frac{\bar{w}-\overline{a_{0}}}{1-a_{0} \bar{w}}=\sum_{l=1}^{4}\left(\alpha_{l}+\frac{\beta_{l}}{1-\overline{a_{0}} z}\right)\left(\overline{\alpha_{l}}+\frac{\overline{\beta_{l}}}{1-a_{0} \bar{w}}\right)\left(1-z^{2} \bar{w}^{2}\right) \\
+\int_{C} c(\psi) \frac{1-\overline{a_{\psi}} z}{1-\overline{a_{0}} z} \frac{1-a_{\psi} \bar{w}}{1-a_{0} \bar{w}}\left(1-z^{2} \bar{w}^{2} \frac{z-a_{\psi}}{1-\overline{a_{\psi}} z} \frac{\bar{w}-\overline{a_{\psi}}}{1-a_{\psi} \bar{w}}\right) d \mu(\psi) .
\end{array}
$$

Multiplying both sides by $\left(1-\overline{a_{0}} z\right)\left(1-a_{0} \bar{w}\right)$ we get

$$
\begin{aligned}
\left(1-\overline{a_{0}} z\right)(1 & \left.-a_{0} \bar{w}\right)-z^{2} \bar{w}^{2}\left(z-a_{0}\right)\left(\bar{w}-\overline{a_{0}}\right) \\
= & \sum_{l=1}^{4}\left(\alpha_{l}\left(1-\overline{a_{0}} z\right)+\beta_{l}\right)\left(\overline{\alpha_{l}}\left(1-a_{0} \bar{w}\right)+\overline{\beta_{l}}\right)\left(1-z^{2} \bar{w}^{2}\right) \\
& +\int_{C} c(\psi)\left(\left(1-\overline{a_{\psi}} z\right)\left(1-a_{\psi} \bar{w}\right)-z^{2} \bar{w}^{2}\left(z-a_{\psi}\right)\left(\bar{w}-\overline{a_{\psi}}\right)\right) d \mu(\psi),
\end{aligned}
$$

which expands to

$$
\begin{aligned}
1-\overline{a_{0}} z-a_{0} \bar{w}+\left|a_{0}\right|^{2} z \bar{w}-z^{3} \bar{w}^{3}+a_{0} z^{2} \bar{w}^{3}+\overline{a_{0}} z^{3} \bar{w}^{2}-\left|a_{0}\right|^{2} z^{2} \bar{w}^{2} \\
=\sum_{l=1}^{4}\left\{\begin{array}{c}
\left|\alpha_{l}+\beta_{l}\right|^{2}-\left|\alpha_{l}+\beta_{l}\right|^{2} z^{2} \bar{w}^{2} \\
-\left(\alpha_{l}+\beta_{l}\right) \overline{\alpha_{l}} a_{0} \bar{w}+\left(\alpha_{l}+\beta_{l}\right) \overline{\alpha_{l}} a_{0} z^{2} \bar{w}^{3} \\
-\left(\overline{\alpha_{l}}+\overline{\beta_{l}}\right) \alpha_{l} \overline{a_{0}} z+\left(\overline{\alpha_{l}}+\overline{\beta_{l}}\right) \alpha_{l} \overline{a_{0}} z^{3} \bar{w}^{2} \\
+\left|\alpha_{l}\right|^{2}\left|a_{0}\right|^{2} z \bar{w}-\left|\alpha_{l}\right|^{2}\left|a_{0}\right|^{2} z^{3} \bar{w}^{3}
\end{array}\right. \\
+\int_{C} c(\psi)\left[\begin{array}{c}
1-\overline{a_{\psi}} z-a_{\psi} \bar{w}+\left|a_{\psi}\right|^{2} z \bar{w} \\
-z^{3} \bar{w}^{3}+\overline{a_{\psi}} z^{3} \bar{w}^{2}+a_{\psi} z^{2} \bar{w}^{3}-\left|a_{\psi}\right|^{2} z^{2} \bar{w}^{2}
\end{array}\right] d \mu(\psi) .
\end{aligned}
$$

To get a contradiction, we look at the $\bar{w}, z^{3} \bar{w}^{3}$ and $z^{2} \bar{w}^{2}$ coefficients of this equation. These yield

$$
\begin{aligned}
a_{0} & =\sum_{l=1}^{4}\left(\alpha_{l}+\beta_{l}\right) \overline{\alpha_{l}} a_{0}+\int_{C} c(\psi) a_{\psi} d \mu(\psi), \\
1 & =\sum_{l=1}^{4}\left|\alpha_{l}\right|^{2}\left|a_{0}\right|^{2}+\int_{C} c(\psi) d \mu(\psi), \\
\left|a_{0}\right|^{2} & =\sum_{l=1}^{4}\left|\alpha_{l}+\beta_{l}\right|^{2}+\int_{C} c(\psi)\left|a_{\psi}\right|^{2} .
\end{aligned}
$$

We can easily see that (3.6) implies

$$
\left|a_{0}\right|^{2}=\left|\sum_{l=1}^{4}\left(\alpha_{l}+\beta_{l}\right) \overline{\alpha_{l}} a_{0}+\int_{C} c(\psi) a_{\psi} d \mu(\psi)\right|^{2},
$$

and if we define a Hilbert space $H=\mathbb{C}^{4} \oplus L^{2}(\mu)$, this can be rewritten as

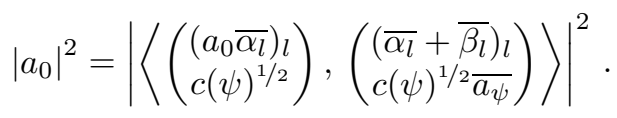


By the Cauchy-Schwarz inequality,

$$
\left|a_{0}\right|^{2} \leq\left(\sum_{l=1}^{4}\left|\alpha_{l}\right|^{2}\left|a_{0}\right|^{2}+\int_{C} c(\psi) d \mu(\psi)\right)\left(\sum_{l=1}^{4}\left|\alpha_{l}+\beta_{l}\right|^{2}+\int_{C} c(\psi)\left|a_{\psi}\right|^{2}\right),
$$

which is in fact an equality by (3.7) and (3.8).

However, as $\overline{a_{\psi}}$ is nonconstant as a function of $\psi, c(\psi)^{1 / 2}$ and $c(\psi)^{1 / 2} \overline{a_{\psi}}$ are linearly independent, implying the inequality must be strict, and so giving a contradiction.

Remark 10. The last part of the proof is a slight oversimplification. If $c(\psi)$ is nonzero at exactly one point $\psi$ (and that $\psi$ is singular with respect to $\mu$ ), then this linear independence argument does not hold. However, a simple calculation by equating coefficients (which is omitted) shows that if the vectors are linearly dependent, so $c(\psi)$ is nonzero at exactly one point, then that point must be $\psi_{0} \notin C$, which is also a contradiction.

Remark 11. In our view, the proof is not entirely satisfactory. It would seem more natural to use the fact that the Agler-Herglotz representation from Theorem 6 was parametrized by extreme measures, although there is no obvious way to do this.

Remark 12. The fact that the minimum set of test functions is parametrized by the sphere is striking, as the set of kernels given in [DPRS07] is also parametrized by the sphere, and conjectured to be minimal (that paper contains some partial results, towards this aim).

Similarly, Abrahamse gave (in Abr79]) a set of kernels corresponding to interpolation on a multiply connected ( $n$-holed) domain, parametrized by the $n$-torus, and conjectured that this set of kernels was minimal (there are some partial results in this direction in [BC96]); in [DM07] and [Pic10, the authors give sets of test functions for $n$-holed domains, which are also parametrized by the $n$-torus, and are minimal. While the main aim of [Pic10] was not to investigate test functions, Note 2.10 of Pic10 gives the set of test functions, and the results of Section 5.2 of Pic10 are broadly analogous to the results used in the proof of Theorem 9 in this paper, as well as those in Proposition 5.3 of [DM07. The test functions in [DM07] and Pic10, are based on a construction given in DM05.

Perhaps there is some sort of duality between minimal sets of test functions and minimal sets of kernels? A possible counterexample to such a duality may occur with the bidisk; it is well known (see for example AM02]) that only two test functions are needed for the bidisk, whereas in [MP02, the authors conjecture that infinitely many kernels are required.

3.7. Generalization. We will briefly discuss how these ideas could be generalized to other spaces. In $\operatorname{Rag} 08$, Raghupathi looks at spaces of the form $\mathbb{C}+B H^{\infty}$ for a Blaschke product $B$. These spaces are a natural generalization of the space $H_{1}^{\infty}$, and the author provides a generalization of the first form of the Nevanlinna-Pick theorem from DPRS07 (a generalization of the second form is given in BBT08).

We used the Herglotz representation to turn a linear equation $\left(f^{\prime}(0)=0\right)$ into a constraint on probability measures (that they have zero mean), found the extreme points of the set of constrained probability measures, and then used those extreme probability measures to generate our test functions.

Suppose we want to apply these techniques to $\mathbb{C}+B H^{\infty}$. It is fairly clear that we can come up with a set of linear equations that all functions in $\mathbb{C}+B H^{\infty}$ must 
satisfy (functions must be constant at zeroes of $B$, and have a prescribed number of zero derivatives at repeated zeroes of $B$ ). It should also be fairly easy to turn this set of linear equations into a set of constraints on probability measures.

The difficulty comes when we calculate extreme points. Theorem 5 should work well enough, so if we have $n$ independent equations, we can say that extreme measures are supported on at most $2 n+1$ points. However, the reasoning that followed Theorem 5 (which showed precisely which such measures would give functions in $H_{1}^{\infty}$ ) relied on a geometric interpretation of the probability constraint, which does not obviously generalize to other types of constraint.

If we could calculate the extreme measures, these should generate test functions in precisely the manner presented here. However, the proof that the test functions we have found are minimal is heavily dependent — perhaps overly dependent - on explicit calculations using the test functions, and such calculations would no doubt become far more complex.

\section{REFERENCES}

[Abr79] Marine B. Abrahamse, The Pick interpolation theorem for finitely connected domains, Michigan Math. J. 26 (1979), no. 2, 195-203. MR532320 (80j:30052)

[AHR08] Jim Agler, John Harland, and Benjamin J. Raphael, Classical function theory, operator dilation theory, and machine computation on multiply-connected domains, Memoirs of the American Mathematical Society 191 (2008), no. 892, viii+159. MR2375060 (2009d:47011)

[AM02] Jim Agler and John E. McCarthy, Pick interpolation and Hilbert function spaces, Graduate Studies in Mathematics, American Mathematical Society, 2002. MR.1882259 (2003b:47001)

[BBT08] Joseph A. Ball, Vladimir Bolotnikov, and Sanne Ter Horst, A constrained NevanlinnaPick interpolation problem for matrix-valued functions, ArXiv: 0809.2345, September 2008.

[BC96] Joseph A. Ball and Kevin F. Clancey, Reproducing kernels for Hardy spaces on multiply connected domains, Integral Equations and Operator Theory 25 (1996), 35-57. MR.1386327 (97f:46042)

[DM05] Michael A. Dritschel and Scott McCullough, The failure of rational dilation on a triply connected domain, Journal of The American Mathematical Society 18 (2005), no. 4, 873-918. MR2163865 (2008i:47024)

[DM07] _ Test functions, kernels, realizations and interpolation, Operator Theory, Structured Matrices and Dilations: Tiberiu Constantinescu Memorial Volume, pp. 153179, Theta Foundation, Bucharest, 2007. MR2389623 (2008k:47033)

[DPRS07] Kenneth R. Davidson, Vern I. Paulsen, Mrinal Raghupathi, and Dinesh Singh, A constrained Nevanlinna-Pick interpolation problem, Indiana University Mathematics Journal 58 (2009), no. 2, 709-732. MR2514385 (2010j:30077)

[MP02] Scott McCullough and Vern Paulsen, $C^{*}$-envelopes and interpolation theory, Indiana University Mathematics Journal 51 (2002), no. 2, 479-505. MR.1909298(2003e:46086)

[Pic10] James Pickering, Counterexamples to rational dilation on symmetric multiply connected domains, Complex Analysis and Operator Theory 4 (2010), no. 1, 55-95. MR2643788

[Rag08] Mrinal Raghupathi, Nevanlinna-Pick interpolation for $\mathbb{C}+B H^{\infty}$, Integral Equations and Operator Theory 63 (2009), 103-125. MR2480640 (2010f:47033)

Department of Mathematics, University of Newcastle-upon-Tyne, NewCastle-uponTyne, NE1 7RU, United Kingdom

E-mail address: m.a.dritschel@ncl.ac.uk

Department of Mathematics, University of NewCastle-upon-Tyne, NewCastle-uponTyne, NE1 7RU, United Kingdom

E-mail address: james.pickering@ncl.ac.uk 